\title{
A propos d'un cas de gastro-entérite fébrile due à une anguillulose chez une patiente gabonaise traitée pour un lupus
}

\author{
About a Case of Febrile Gastroenteritis Due to Strongyloidiasis in a Gabonese Patient Treated \\ for Lupus
}

\author{
S. Ntsame Ngoua $\cdot$ J. Iba-Ba $\cdot$ M. Igala $\cdot$ P.E. Itoudi Bignoumba $\cdot$ L. Pemba $\cdot$ M.I.C. Nziengui Madjinou \\ L. Ledaga $\cdot$ L. Missounga $\cdot$ D. Malekou $\cdot$ J.B. Boguikouma
}

Reçu le 3 octobre 2018; accepté le 22 février 2019

(C) Société de pathologie exotique et Lavoisier SAS 2019

\begin{abstract}
Résumé L'anguillulose peut parfois être source d'errance diagnostique chez un patient porteur d'une maladie autoimmune vivant en milieu tropical, et ce malgré un déparasitage systématique par l'albendazole ( $40 \mathrm{mg} /$ jour / 3 jours), avant la mise en route d'une corticothérapie. Nous rapportons une observation de gastroentérite fébrile, compliquée de signes de déshydratation intra et extracellulaire, chez une patiente lupique de 37 ans, dont les biopsies duodénales, et la parasitologie des selles ont conduit au diagnostic d'anguillulose traitée efficacement par ivermectine per os (2 doses de 200 microgrammes $/ \mathrm{kg}$, en prise unique à 2 semaines d'intervalle l'une de l'autre). Ce traitement faisait suite à l'échec d'un premier traitement par 5 jours d'albendazole (400 mg/jour).
\end{abstract}

Keywords Gastroenteritis - Lupus · Corticosteroids · Strongyloidiasis - Ivermectin - Albendazole · Hospital · Libreville $\cdot$ Gabon $\cdot$ Sub-Saharan Africa

\begin{abstract}
Srongyloidiasis can sometimes be a source of diagnostic wandering in a patient with an autoimmune disease living in a tropical environment, despite systematic deworming with albendazole $(400 \mathrm{mg} /$ day $/ 3$ days $)$, prior to the starting of a corticotherapy. We report an observation of a febrile gastroenteritis complicated by signs of intra and extracellular dehydration, in a 37-year-old lupus patient, including duodenal biopsies, and stool parasitology, which led to the diagnosis of strongyloidiasis effectively treated by ivermectin per os (two doses) of 200 micrograms $/ \mathrm{kg}$, once every 2 weeks apart), following failure of a first 5-days course of albendazole ( $400 \mathrm{mg} /$ day).
\end{abstract}

S. Ntsame Ngoua $(\bowtie) \cdot J$. Iba-Ba $\cdot$ M. Igala

P.E. Itoudi Bignoumba · L. Pemba · M.I.C. Nziengui Madjinou

L. Ledaga $\cdot$ L. Missounga $\cdot$ D. Malekou $\cdot$ J.B. Boguikouma

CHU de Libreville, BP 2228, Gabon

e-mail : blanchenns@yahoo.fr
Mots clés Gastroentérite · Lupus · Corticoïdes ·

Anguillulose · Ivermectine $\cdot$ Albendazole $\cdot$ Hôpital ·

Libreville $\cdot$ Gabon $\cdot$ Afrique intertropicale

\section{Introduction}

Le risque de survenue d'une anguillulose maligne impose de traiter de façon présomptive les patients avant l'initiation d'une corticothérapie lorsque ceux-ci sont susceptibles d'être porteurs d'anguillules et donc, de façon systématique, tous ceux qui vivent en zone subsaharienne. Malgré cela, la vigilance doit rester de mise, comme le montre l'observation que nous relatons, le risque de ré-infection et même d'infection de novo au décours de la corticothérapie ne pouvant être exclu en zone d'endémie, de même que l'échec d'un traitement présomptif par l'albendazole dont l'efficacité est inférieure à celle de l'ivermectine,

\section{Observation}

Il s'agit d'une patiente de 37 ans, résidant en zone côtière et marécageuse, suivie depuis 6 mois pour un lupus systémique traité, après déparasitage systématique par de l'albendazole ( $400 \mathrm{mg}$ /jour / 3 jours), par prednisone $50 \mathrm{mg}$ et hydroxychloroquine ( $400 \mathrm{mg} / \mathrm{jour})$ per os. Cette patiente s'est présentée en consultation pour gastro-entérite fébrile $38^{\circ} 2 \mathrm{C}$, marquée par des vomissements post prandiaux précoces et des selles diarrhéiques sans modification de leur coloration, compliquée de déshydratation mixte avec sensibilité de l'épigastre, sans signe d'irritation péritonéale.

Il existait une hyperleucocytose à $10300 / \mathrm{mm}^{3}$ à prédominance neutrophile et sans éosinophilie, une anémie microcytaire à 10,2 $\mathrm{g} / \mathrm{dl}$, des plaquettes à 495000 , une vitesse de sédimentation à $60 \mathrm{~mm}$, une C-réactive protéine à $96 \mathrm{mg} / \mathrm{dl}$, 
une bandelette urinaire à 3 croix pour les nitrites sans protéinurie ni hématurie, et une perturbation du bilan rénal avec créatininémie à $170 \mu \mathrm{mol} / 1$ et urémie à $10,2 \mathrm{mmol} / 1$. La persistance des vomissements, malgré un traitement associant réhydratation, anti-émétique et levofloxacine intraveineux (500 mg/jour), a alors conduit à exclure une grossesse par le dosage des béta HCG plasmatiques, à réaliser une tomodensitométrie cérébrale qui s'est avérée normale et une endoscopie digestive haute mettant en évidence une gastroduodénite érosive et congestive, avec à l'histologie des biopsies gastriques et duodénales, une gastrite chronique avec infiltration de la muqueuse duodénale par des larves de Strongylö̈des stercoralis, également retrouvées dans les selles. La coproculture n'a pu été réalisée pour éliminer une infection bactérienne associée.

Devant l'inefficacité d'un premier traitement par albendazole ( $400 \mathrm{mg} /$ jour pendant 5 jours) sur les vomissements, un second traitement par ivermectine per os $(2$ doses uniques de $200 \mu \mathrm{g} / \mathrm{kg}$ à 15 jours d'intervalle) a été administré, permettant l'arrêt des vomissements en 24 heures, de la diarrhée et le retour à la normale de la température en $48 \mathrm{~h}$. Les biopsies gastriques et duodénales de contrôle réalisées à deux semaines de la seconde prise d'ivermectine ont confirmé la disparition totale des larves.

\section{Discussion}

L'anguillulose est une infection parasitaire intestinale du tractus digestif par Strongylö̈des stercoralis, dont le diagnostic est établi par la mise en évidence de larves dans les matières fécales et/ou au niveau de la muqueuse duodénale [4]. Elle sévit dans les régions chaudes et humides d'Afrique tropicale, où elle touche électivement les habitants des zones rurales travaillant sur des terres inondées où des terrains boueux, propices à la prolifération et à la conservation des larves [4]. Une enquête a été réalisée de septembre 2013 à juin 2014, dans trois régions du Gabon, Libreville, la capitale (zone urbaine), Melen (zone semi urbaine) et Dienga (regroupement de villages) : sur 451 personnes interrogées dont les sang et selles ont été prélevés pour la recherche de parasitoses, 3,7 \% étaient positifs pour Strongyloïdes stercolaris [3].

Dans le cas que nous rapportons, le diagnostic a été retardé par l'absence d'éosinophilie : ce qui est d'un grand classique lors d'une corticothérapie. La persistance des vomissements et de la diarrhée malgré une cure d'albendazole aux doses recommandées par son fabricant $(400 \mathrm{mg} /$ jour pendant 5 jours), en a fait rediscuter l'efficacité, et prôner son remplacement par de l'ivermectine qui a permis d'obtenir une disparition des symptômes. Il semblerait qu'une dose de $800 \mathrm{mg}$ au lieu de $400 \mathrm{mg}$ soit bien plus efficace, comme cela a été montré dès 1990 [1], mais cette possibilité de doubler les doses ne figure pas dans les indications du fabricant. Dans l'anguillulose, l'immunodépression de l'hôte (corticothérapie générale, immunosupresseurs, infection chronique par le virus HTLV-I, etc.) [2] favorise le cycle d'auto-infestation qui peut s'emballer et aboutir à une anguillulose qualifiée de maligne lorsque l'atteinte est polyviscérale, notamment neurologique, mettant en jeu le pronostic vital. Il est donc de règle dans les régions où l'anguillulose est endémique, de traiter systématiquement contre ce parasite toute personne devant être mise sous corticoïdes. La patiente dont nous rapportons le cas était immunodéprimée par le lupus et les thérapeutiques immunosuppressives utilisées pour le contrôle de cette affection. Compte tenu du fait qu'elle vivait dans une zone côtière marécageuse, donc un écosystème propice aux ré-infections ou à une infection de novo, il aurait certainement été souhaitable, bien qu'irréaliste, d'envisager un changement du lieu de résidence à défaut d'un assainissement de la zone concernée.

\section{Conclusion}

Chez les patients immunodéprimés vivant en milieu tropical, et sous corticothérapie au long cours, il est souhaitable de rechercher une anguillulose et, quels que soient les résultats de cette recherche, de prescrire par sécurité un traitement présomptif. Les risques de ré-infestation doivent être également pris en compte lorsque la corticothérapie est de longue durée, le milieu favorable à la survie et à la prolifération des larves, les occupations et les comportements favorables aux contacts avec les sols humides et boueux. L'anthelmintique utilisé doit être, par mesure de sécurité, l'ivermectine.

Liens d'intérêt : Les auteurs déclarent ne pas avoir de liens d'intérêt.

\section{Références}

1. Coulaud JP (1990) Le traitement de l'anguillulose en 1990. Méd Afr Noire 37(10):600-4

2. Gentilini M, Caumes E, Danis M, et al (2012) Anguillulose. In : Médecine Tropicale 6ème édition, Médecines Sciences, Lavoisier, Paris, pp 312-6

3. M'bondoukwé NP, Kendjo E, Mawili-Mboumba DP, et al (2018) Prevalence of and risk factors for malaria, filariasis, and intestinal parasites as single infections or co-infections in different settlements of Gabon, Central Africa. Infect Dis Poverty 7(1):6. doi: 10.1186/s40249-017-0381-4

4. Zaha O, Hirata T, Kinjo F, Saito A (2000). Strongyloidiasis - progress in diagnosis and treatment. ntern Med 39(9):695-700 